\title{
Biegezugverstärkung von BS-Holz mit CFK- und AFK-Lamellen
}

\section{H.J. Blaß, M. Romani}

Das Ziel der dargestellten Untersuchung war, Angaben zum Trag- und Verformungsverhalten von Verbundträgern aus Brettschichtholz (BS-Holz) und unidirektional verstärkten Faserverbundkunststoffen (FVK) zu erhalten. Dazu wurden der Einfluss des Verstärkungsgrades, der geometrischen Anordnung der Verstärkung und der Einfluss von CFK-Lamellen und AFK-Lamellen genauer untersucht und verstärkte BS-Holz Träger unter praxisnahen Bedingungen hergestellt.

\section{Load carrying behaviour of composite glued laminated timber beams reinforced with fibre reinforced plastic}

The aim of the investigation was to study the load carrying and deformation behaviour of composite glued laminated timber beams reinforced with fibre reinforced plastic (FRP). For this purpose the influence of the amount and position of the reinforcement and of different FRP laminations and adhesives was determined. Reinforced beams were produced under practice conditions.

\section{Einleitung ${ }^{1}$}

Etwa seit dem Beginn des 20. Jahrhunderts wurden unter anderem von Otto Hetzer Holzbalken durch Verklebung einzelner Bohlen hergestellt. Diese Bauweise gilt als Vorläufer der Brettschichtholzbauweise. Spätere Untersuchungen zeigten, dass die Verklebung einzelner Bretter zu BS-Holz zu geringeren Streuungen der Materialeigenschaften durch das gleichmäßige Verteilen der Wuchsunregelmäßigkeiten und damit zu einer Erhöhung der Tragfähigkeit des BSHolzes im Vergleich zu Vollholz führt.

Die Tragfähigkeit des BS-Holzes wird durch die mechanischen Eigenschaften des Holzes und durch die Festigkeit der Keilzinkenverbindungen bestimmt. So

Information:

Prof Dr.-Ing. H. J. Blaß, Dipl.-Ing. M. Romani

Universität Karlsruhe $(\mathrm{TH})$

Lehrstuhl für Ingenieurholzbau und Baukonstruktionen

Ernst Gaber-Straße

Geb. 10.50, 76128 Karlsruhe

1 Die hier dargestellten Versuche wurden in den Jahren 1998 bis 2000 im Auftrag der DGfH durchgeführt und aus Haushaltsmitteln des Bundesministeriums für Wirtschaft und Technologie (BMWi) über die Arbeitsgemeinschaft industrieller Forschungsvereinigungen „Otto von Guerike“ e.V. (AiF) gefördert. 
versagen BS-Holz-Träger unter einer Momentenbeanspruchung hauptsächlich im Zugbereich in der Nähe von Wuchsunregelmäßigkeiten oder an den Keilzinkenverbindungen (KZV). Steigerungen der Tragfähigkeit von BS-Holz können durch die Verstärkung der Wuchsunregelmäßigkeiten mit Werkstoffen hoher Festigkeit und hoher Steifigkeit erzielt werden. Dies sind z. B. Laminate aus Kunststofffasern wie Aramid oder Kohlenstoff.

Neben sehr guten Festigkeits- und Steifigkeitseigenschaften bieten Faserverbundkunststoffe (FVK) diverse Vorteile für eine gezielte Verstärkung von BS-Holz Trägern im Ingenieurholzbau. So kann das plastische Potential des BS-Holzes bei Biegeträgern durch die Entlastung von Wuchsunregelmäßigkeiten in der Zugzone besser als bisher ausgenutzt werden. Durch die Verstärkung mit FVK-Lamellen wird gleichzeitig die Biegesteifigkeit erhöht, die häufig das maßgebende Bemessungskriterium von Biegeträgern im Ingenieurholzbau darstellt. Aufgrund des Verstärkungseffektes können Bretter der Sortierklasse S7 oder MS7, die bisher nicht für die Herstellung von BS-Holz verwendet wurden, wirtschaftlich eingesetzt werden.

Die genannten Vorteile ermöglichen eine bessere Ausnutzung des Brettangebotes niedriger Sortierklassen und die Dimensionierung mit kleineren Querschnitten bei gleicher Traglast. Hieraus ergeben sich günstigere Transport- und Einbaubedingungen und ein geringeres Gewicht der Tragkonstruktion. Im Bereich der Sanierung und Nutzungsänderung von Tragwerken ist aufgrund der einfachen Applikation der FVK-Lamellen eine nachträgliche Verstärkung wirtschaftlich möglich. Um das Trag- und Verformungsverhalten dieser Bauteile zu untersuchen, wurden von Blaß et al. (2000) an der Versuchsanstalt für Stahl, Holz und Steine, Abt. Ingenieurholzbau der Universität Karlsruhe (TH) Versuche an 38 verstärkten Trägern durchgeführt.

\section{Versuchsmaterial}

\subsection{Brettmaterial}

Für die Bauteilversuche wurde Holz aus maschinell sortierten Brettern der Sortierklassen MS7, MS10 und MS17 ausgewählt. Bei der Auswahl von Brettern der Sortierklasse MS10 wurden die Bretter mit den niedrigsten Elastizitätsmoduln und Rohdichten des jeweils vorhandenen Brettangebotes ausgewählt. Dies geschah, um einen möglicht großen Unterschied in den Eigenschaften des Holzes im Verhältnis zu den Eigenschaften der verwendeten FVK-Lamellen zu erhalten und die Verwendbarkeit von Brettern minderer Qualität für die Herstellung von BS-Holz auf zu zeigen. Der Grundaufbau aller Versuchsträger bestand aus Brettern der Sortierklasse MS10, die zur Ober- bzw. Unterkante des Querschnitte immer geringere Elastizitätsmoduln und Rohdichten aufwiesen.

\subsection{Faserverbundkunststoff Lamellen (FVK-Lamellen)}

Für die Untersuchungen standen zunächst vier verschiedene FVK-Lamellen zur Verfügung, von denen drei für die Bauteilversuche verwendet wurden. Drei der vier FVK-Lamellen bestanden aus Kohlenstofffasern (CF) und eine aus Aramidfasern 
(AF). In Tabelle 1 und Tabelle 2 werden die Bezeichnungen und Eigenschaften der FVK-Lamellen entsprechend den Herstellerangaben und aus Zugversuchen beschrieben. Die AFK-Lamelle L3 war werksseitig auf beiden Seiten, die CFKLamellen L1, L2 und L4 werksseitig auf einer Seite angeschliffen.

Tabelle 1: Vorhandene FVK-Lamellen

Table 1: Available FRP Lamellae

\begin{tabular}{|c|c|c|c|}
\hline Abk. & Produktbezeichnung & Hersteller / Vertrieb & Typ \\
\hline L1 & Sika CarboDur S & Sika Chemie GmbH & CFK \\
\hline L2 & Sika CarboDur H & Sika Chemie GmbH & CFK \\
\hline L3 & - & Akzo Nobel Faser AG & AFK \\
\hline L4 & S\&P CFK Lamelle & Ispo GmbH & CFK \\
\hline
\end{tabular}

Für die Bauteilversuche wurden die zwei CFK-Lamellen L1, L4 und die AFK-Lamelle L3 verwendet.

Tabelle 2: Materialangaben der FVK-Lamellen

Table 2: Material data of the FRP Lamellae

\begin{tabular}{|c|c|c|c|c|}
\hline Abk. & $\begin{array}{c}\text { Zug-E-Modul }{ }^{1)} \\
\text { Mittelwert } \\
{\left[\mathrm{N} / \mathrm{mm}^{2}\right]}\end{array}$ & $\begin{array}{c}\text { Zugfestigkeit }^{1)} \\
\text { Mittelwert } \\
{\left[\mathrm{N} / \mathrm{mm}^{2}\right]}\end{array}$ & Dicke & Breite \\
{$[\mathrm{mm}]$} & {$[\mathrm{mm}]$} \\
\hline L1 & 173.000 & 3.050 & 1,2 & 100 \\
\hline L2 & 304.000 & 1.680 & 1,4 & 50 \\
\hline L3 & 74.000 & 995 & 1,8 & 132 \\
\hline L4 & 199.000 & 2.570 & 1,4 & 100 \\
\hline
\end{tabular}

1) aus Zugversuchen an $50 \mathrm{~mm}$ breiten Proben, Versuchsanzahl 5

\subsection{Klebstoffe}

Als Klebstoffe standen zwei Epoxidharzklebstoffe, die vom Hersteller für eine Verwendung mit den Lamellen L1 und L2 bzw. L4 vorgesehen sind und zwei Klebstoffe aus der BS-Holz Herstellung zur Verfügung. In Tabelle 3 sind die Bezeichnungen dieser Klebstoffe angegeben.

Die Epoxidharzklebstoffe K1 und K2 wurden aufgrund der bewährten Verklebung von CFK-Lamellen im Massivbau ausgewählt. Der Resorcinharzklebstoff K4 wurde gewählt, da eine Verklebung mit Resorcinharzklebstoffen mit der AFK-Lamelle L3 möglich erschien, und dieser Klebstoff bei BS-Holz Herstellern Verwendung findet. Der Polyurethanklebstoff K3 wurde in das Versuchsprogramm aufgenommen, da dieser Klebstoff sowohl bei BS-Holz Herstellern Verwendung findet und gleichzeitig, 
neben der Verklebung der AFK-Lamelle L3, eine Verklebung mit CFK-Lamellen aufgrund von Vorversuchen aussichtsreich erschien.

Tabelle 3: Vorhandene Klebstoffe

Table 3: Available adhesives

\begin{tabular}{|c|c|c|}
\hline Abk. & Produktbezeichnung & Hersteller / Vertrieb \\
\hline K1 & Sikadur-30 & Sika Chemie GmbH \\
\hline K2 & ispo Concretin SK 41 & ispo GmbH \\
\hline K3 & Collano Purbond HB 110 & Ebnöther AG \\
\hline K4 & $\begin{array}{r}\text { Dynosol S-199 } \\
\text { mit Härter H-629 }\end{array}$ & Dyno Industries A.S. \\
\hline
\end{tabular}

Aufgrund der Voruntersuchung wurden für die Bauteilversuche mit einer Herstellung im Labor der Klebstoff K2 und bei der praxisnahen Herstellung bei einem BS-Holz Hersteller der Klebstoff K3 verwendet. Der Epoxidharzklebstoff K2 wurde für die Laborherstellung ausgewählt, da eine tragfähige Verklebbarkeit zwischen den Fügeteilpartnern Holz und FVK erwartet wurde und die Verarbeitbarkeit des Klebstoffes K2 aufgrund eines geringeren Füllstoffanteiles einfacher war als beim Klebstoff K1.

\section{Bauteilversuche}

\subsection{Aufbau, Herstellung und Prüfung der Versuchsträger}

Da die Prüfkörper der Versuchsreihen Tr-1 und Tr-2 mit Brettlamellen ohne KZV hergestellt werden sollten, wurde für alle Träger eine Spannweite von 4,20 m bei einer Gesamtlänge von 4,50 m aufgrund der beschränkten Länge der Einzelbretter festgelegt. In Tabelle 4 sind die durchgeführten Versuchsreihen dargestellt. Als Ausgangsmaterial für die Bauteilversuche wurde für alle Versuchsreihen MS10 und für die Versuchsreihen Tr-1 und Tr-2 im Randbereich bei den äußersten zwei Holzlamellen auch MS7 verwendet. Bei den Versuchsreihen Tr-5 und Tr-6 wurden im Randbereich der Zugzone MS17 Brettlamellen angeordnet. In Tabelle 5 ist der mittlere Längsschwingungs-E-Modul und die mittlere Rohdichte für den Randbereich und den Gesamtquerschnitt angegeben. Der Aufbau der Versuchsträger ist in Bild 1 dargestellt. 


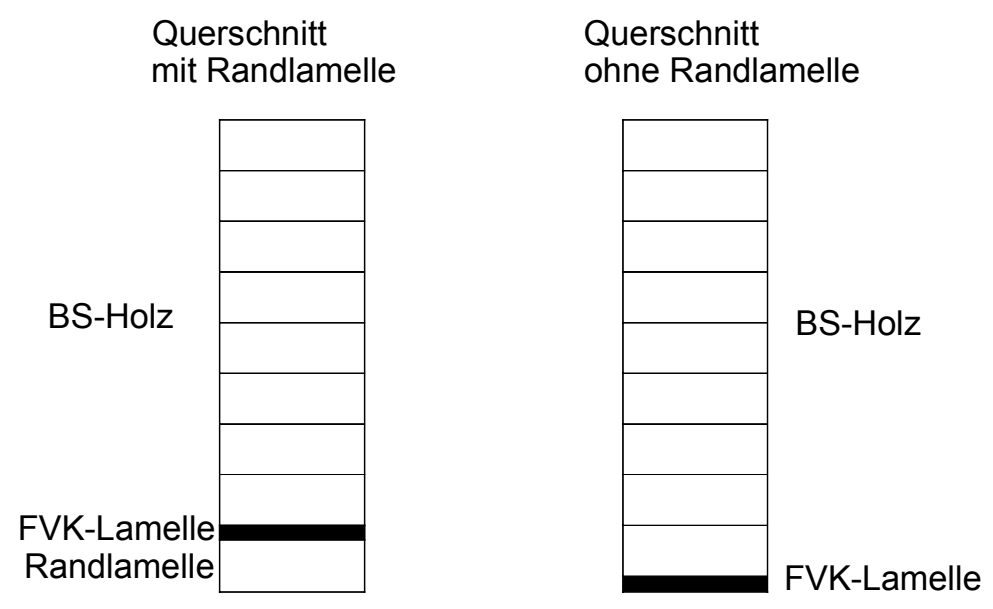

Bild 1: Bezeichnungen der Querschnittsteile und Aufbau der Versuchsträger

Fig. 1: Description of the cross section lay-up of the test girders

Aufgrund der geringen Ausbeute an MS7 wurden MS7 Bretter nur bei den ersten beiden Versuchsreihen im Randbereich der Bauteile verwendet. Bei den übrigen Versuchsreihen wurde auf MS7 Brettlamellen verzichtet und dafür MS10 Brettlamellen mit möglichst niedrigem Elastizitätsmodul und niedriger Rohdichte im Randbereich angeordnet. Das verwendete Brettangebot hatte mit einem mittleren Längsschwingungs-E-Modul von $9800 \mathrm{~N} / \mathrm{mm}^{2}$ einen vergleichsweise geringen Elastizitätsmodul für MS10 Bretter.

Tabelle 4: Versuchsprogramm der Bauteilversuche

Table 4: Test programme of the beam tests

\begin{tabular}{|c|c|c|c|c|c|c|c|}
\hline Reihe & $\begin{array}{c}\text { Anzahl } \\
\text { der } \\
\text { Versuche }\end{array}$ & $\begin{array}{c}\text { Sortierklasse } \\
\text { der } \\
\text { Einzelbretter }\end{array}$ & $\begin{array}{c}\text { Randlamelle } \\
\text { aus } \\
\text { Holz }\end{array}$ & \begin{tabular}{|c|} 
Mittlere \\
Höhe/Breite \\
[mm]
\end{tabular} & $\begin{array}{c}\text { FVK- } \\
\text { Lamelle } \\
\text { (Anzahl) }\end{array}$ & $\begin{array}{c}\text { Kleb- } \\
\text { stoff }\end{array}$ & KZV \\
\hline Tr-1 & 5 & MS7 / MS10 & MS7 / MS10 & $308 / 100$ & L1 (1) & $\mathrm{K} 2$ & ohne \\
\hline Tr-2 & 5 & MS7 / MS10 & MS7 / MS10 & $312 / 100$ & L4 (2) & K2 & ohne \\
\hline Tr-3 & 5 & MS10 & MS10 & $308 / 100$ & L1 (1) & $\mathrm{K} 2$ & mit \\
\hline Tr-4 & 5 & MS10 & MS10 & $312 / 100$ & L4 (2) & $\mathrm{K} 2$ & mit \\
\hline Tr-5 & 5 & MS10 / MS17 & MS17 & $312 / 100$ & L3 (4) & K3 & mit \\
\hline $\operatorname{Tr}-6$ & 5 & MS10 / MS17 & MS10 & $312 / 100$ & L3 (4) & K3 & mit \\
\hline Tr-7 & 5 & MS10 & - & $308 / 100$ & L1 (1) & K3 & mit \\
\hline Tr-8 & 3 & MS10 & - & $310 / 100$ & L4 (2) & K3 & mit \\
\hline
\end{tabular}

Die Keilzinkenverbindungen wurden versetzt im Bereich zwischen den Lasteintragungspunkten angeordnet. Hierbei wurden die oberen zwei und die unteren drei Brettlamellen mit einer Keilzinkenverbindung versehen. Die FVK-Lamellen wurden über die gesamte Länge und Breite der Träger angeordnet. 
Tabelle 5: Längsschwingungs-E-Modul und Rohdichte bei der jeweiligen Feuchte der Einzelbretter

Table 5: Longitudinal vibration modulus of elasticity and density of the single boards

\begin{tabular}{|c|c|c|c|c|c|}
\hline \multirow{2}{*}{\multicolumn{2}{|c|}{ Reihe }} & \multicolumn{2}{|c|}{ E-Modul } & \multicolumn{2}{|c|}{ Rohdichte } \\
\hline & & $\begin{array}{c}\text { Mittelwert } \\
{\left[\mathrm{N} / \mathrm{mm}^{2}\right]}\end{array}$ & $\begin{array}{c}\text { Variations.- } \\
\text { koeff. [\%] }\end{array}$ & $\begin{array}{c}\text { Mittelwert } \\
{\left[\mathrm{kg} / \mathrm{m}^{3}\right]}\end{array}$ & $\begin{array}{l}\text { Variations.- } \\
\text { koeff. [\%] }\end{array}$ \\
\hline \multirow[t]{2}{*}{ Tr-1 } & Querschnitt & 9500 & 14,85 & 391 & 5,60 \\
\hline & Randbereich & 9100 & 15,18 & 385 & 5,92 \\
\hline \multirow[t]{2}{*}{$\operatorname{Tr}-2$} & Querschnitt & 9200 & 16,10 & 389 & 6,03 \\
\hline & Randbereich & 8700 & 15,15 & 383 & 5,09 \\
\hline \multirow[t]{2}{*}{ Tr-3 } & Querschnitt & 9600 & 9,48 & 398 & 6,35 \\
\hline & Randbereich & 9300 & 7,57 & 392 & 6,13 \\
\hline \multirow[t]{2}{*}{ Tr-4 } & Querschnitt & 9600 & 9,31 & 395 & 6,12 \\
\hline & Randbereich & 9300 & 7,57 & 393 & 6,06 \\
\hline \multirow[t]{3}{*}{ Tr-5 } & Querschnitt MS10 & 10600 & 5,19 & 421 & 5,78 \\
\hline & Randbereich MS10 & 10100 & 5,45 & 412 & 5,90 \\
\hline & Lamellen mit MS17 & 15400 & 9,72 & 491 & 8,67 \\
\hline \multirow[t]{3}{*}{$\operatorname{Tr}-6$} & Querschnitt MS10 & 10600 & 9,25 & 418 & 7,19 \\
\hline & Randbereich MS10 & 10000 & 6,06 & 410 & 5,99 \\
\hline & Lamelle mit MS17 & 15400 & 9,72 & 491 & 8,67 \\
\hline \multirow[t]{2}{*}{$\operatorname{Tr}-7$} & Querschnitt & 9700 & 7,77 & 401 & 6,49 \\
\hline & Randbereich & 9300 & 6,15 & 399 & 6,88 \\
\hline \multirow[t]{2}{*}{ Tr-8 } & Querschnitt & 9700 & 8,16 & 399 & 6,51 \\
\hline & Randbereich & 9300 & 8,54 & 396 & 6,63 \\
\hline \multirow[t]{2}{*}{ Tr-1 bis Tr-8 } & Querschnitt MS10 & 9800 & 11,24 & 402 & 6,93 \\
\hline & Randbereich MS10 & 9500 & 9,38 & 398 & 6,61 \\
\hline
\end{tabular}

Randbereich: Zwei obersten Holzlamellen und drei untersten Holzlamellen Querschnitt: Alle Holzlamellen des Querschnittes

Die Versuchsreihen Tr-1 bis Tr-4 mit dem Klebstoff K2 wurden im Labor hergestellt. Der Epoxidharzklebstoff wurde bei diesen Versuchsreihen zur Verklebung zwischen den CFK-Lamellen und zwischen CFK-Lamellen und Holz verwendet. Das Sollmaß der Klebefugendicke mit dem Epoxidharzklebstoff betrug $1 \mathrm{~mm}$. Für die Versuchsreihen Tr-1 bis Tr-4 wurden die Grundträger beim BS-Holz Hersteller vorgefertigt. Die Verstärkungslagen und die Randlamellen wurden im Prüflabor bei Normalklima 20/65 aufgeklebt. Die Verklebung der CFK-Lamellen erfolgte nach dem Anschleifen und Entfetten der jeweiligen Oberfläche der CFKLamelle. 
Die Versuchsreihen Tr-5 bis Tr-8 wurden als komplette Verbundträger bei einem BS-Holz Hersteller praxisnah hergestellt, bei dem der Polyurethanklebstoff K3 in der normalen Herstellung von BS-Holz verwendet wird. Beim Klebstoffauftrag auf die FVK-Lamelle wurde abweichend von der üblichen BS-Holz Herstellung eine Trägerlamelle aus Holz verwendet, auf welcher die FVK-Lamelle lose aufgelegt wurde um die gleiche Gesamthöhe einer normalen Brettlamelle zu erhalten. In Bild 2 ist eine AFK-Lamelle beim Klebstoffauftrag dargestellt.

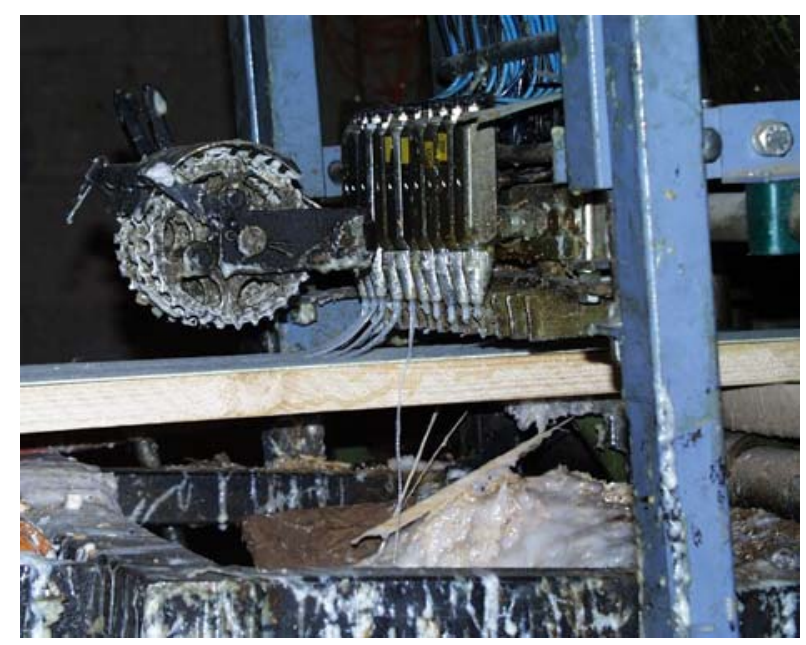

Bild 2: AFK-Lamelle mit Trägerlamelle beim Klebstoffauftrag

Fig. 2: AFK-Lamella with supporting lamella while applying adhesive

Die Bauteilversuche wurden in Anlehnung an DIN EN 408 durchgeführt. Die Verformungsgeschwindigkeit betrug zwischen 15 und $20 \mathrm{~mm} / \mathrm{min}$. Die Versuche wurden an einer $400 \mathrm{kN}$ EPZ-Eigenbau und $100 \mathrm{kN}$ EPZ-Eigenbau durchgeführt. Der Versuchsaufbau ist in Bild 3 dargestellt. Die Verschiebungen wurden in Trägermitte an der Oberkante durch einen induktiven Wegaufnehmer mit einem Nennweg von $200 \mathrm{~mm}$, an den Auflagern beidseitig mit induktiven Wegaufnehmern mit einem Nennweg von $2 \mathrm{~mm}$ sowie im mittleren Bereich mit zwei induktiven Wegaufnehmern mit einem Nennweg von $10 \mathrm{~mm}$, bezogen auf die geometrische Mittellinie des Querschnitts, gemessen.

Die Auswertung des Elastizitätsmoduls nach DIN EN 408 erfolgte mit den gemittelten Werten der Wegaufnehmer W10. Die Gesamtverformung bezogen auf die Spannweite von 4,20 m wurde aus der an der Bauteiloberkante gemessenen Vertikalverformung und unter Berücksichtigung der Eindrückung am Auflager ermittelt. Die Eindrückung am jeweiligen Auflager wurde aus dem Mittelwert der dort befindlichen Wegaufnehmer ermittelt. 


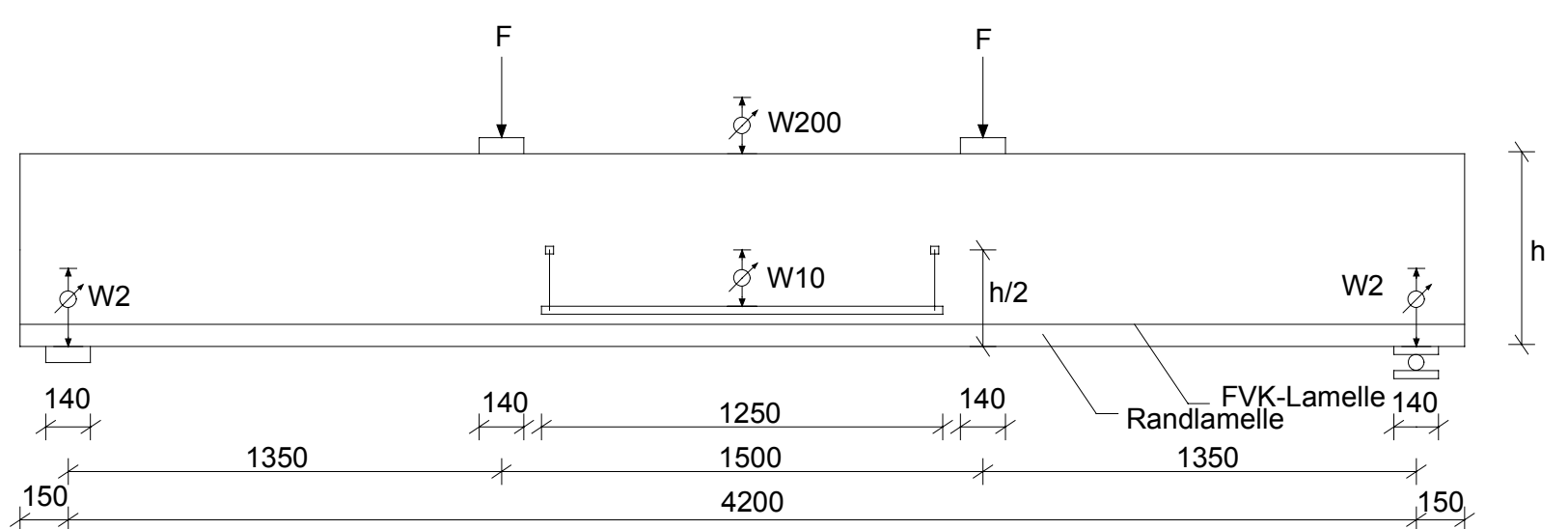

[Maße in mm]

Bild 3: Versuchsaufbau der Bauteilversuche

Fig. 3: Test set-up of the beam tests

\subsection{Ergebnisse und Vergleich der Versuchsreihen}

\subsubsection{Ergebnisse der Bauteilversuche}

Die Ergebnisse der Versuchsreihen sind in der Tabelle 6 zusammen gefasst. Die Abfolge eines typischen Versagens eines Versuchs mit Randlamelle und ohne Randlamelle zeigt Bild 10. Last-Verformungsdiagramme sind in Bild 6 bis Bild 9 dargestellt.

Tabelle 6: Mittelwerte der einzelnen Versuchsreihen

Table 6: Mean values of the single test series

\begin{tabular}{|c|c|c|c|c|c|c|c|c|c|}
\hline & \multicolumn{8}{|c|}{ Mittelwerte der einzelnen Versuchsreihen } \\
\hline & & Tr-1 & $\operatorname{Tr}-2$ & Tr-3 & Tr-4 & Tr-5 & $\operatorname{Tr}-6$ & $\operatorname{Tr}-7$ & Tr-8 \\
\hline \multicolumn{10}{|c|}{ Versagen Randlamelle } \\
\hline $\mathrm{F}_{\text {Randlamelle }}$ & {$[\mathrm{kN}]$} & 37,3 & 42,7 & 39,3 & 47,9 & 53,8 & 52,1 & $49,8^{1)}$ & $66,0^{1)}$ \\
\hline$f_{m}=M / W$ & {$\left[\mathrm{~N} / \mathrm{mm}^{2}\right]$} & 31,8 & 35,5 & 33,5 & 39,9 & 44,8 & 43,4 & 42,5 & 55,7 \\
\hline Variations.-koeff. & [\%] & 14,3 & 16,0 & 6,2 & 6,4 & 18,3 & 5,8 & 5,0 & 6,2 \\
\hline Durchbiegung & {$[\mathrm{mm}]$} & 42,8 & 44,3 & 48,5 & 54,2 & 57,8 & 49,0 & 61,7 & 72,0 \\
\hline Variations.-koeff. & {$[\%]$} & 12,4 & 15,7 & 6,1 & 6,7 & 21,5 & 8,1 & 4,8 & 7,6 \\
\hline $\begin{array}{l}\text { Versagensursache } \\
\text { (Häufigkeit) }\end{array}$ & & A (5) & A (5) & $\begin{array}{l}\text { A (3) } \\
\text { K (2) }\end{array}$ & $\begin{array}{l}\mathrm{A}(3) \\
\mathrm{K}(2)\end{array}$ & $\begin{array}{l}\mathrm{A}(1) \\
\mathrm{K}(4)\end{array}$ & A (5) & $\begin{array}{l}\text { A (2) } \\
\text { K (3) }\end{array}$ & $\begin{array}{l}\mathrm{A}(1) \\
\mathrm{K}(2)\end{array}$ \\
\hline \multicolumn{10}{|l|}{ Gesamtversagen } \\
\hline$F_{\max }$ & {$[\mathrm{kN}]$} & 44,1 & 57,7 & 43,0 & 58,1 & 60,5 & 59,1 & 49,8 & 66,5 \\
\hline$f_{m}=M N$ & {$\left[\mathrm{~N} / \mathrm{mm}^{2}\right]$} & 37,6 & 48,0 & 36,7 & 48,3 & 50,3 & 49,2 & 42,5 & 56,8 \\
\hline Variations.-koeff. & {$[\%]$} & 12,5 & 4,7 & 13,0 & 5,9 & 6,8 & 3,5 & 5,0 & 6,9 \\
\hline Durchbiegung & {$[\mathrm{mm}]$} & 70,2 & 86,6 & 64,0 & 97,5 & 88,6 & 83,2 & 61,7 & 74,8 \\
\hline Variations.-koeff. & {$[\%]$} & 16,1 & 6,5 & 33,4 & 5,8 & 12,1 & 6,6 & 4,8 & 12,3 \\
\hline
\end{tabular}




\begin{tabular}{|c|c|c|c|c|c|c|c|c|}
\hline $\begin{array}{l}\text { Versagensursache } \\
\text { (Häufigkeit) }\end{array}$ & $A(5)$ & $A(5)$ & $\begin{array}{l}\mathrm{A}(3) \\
\mathrm{K}(2)\end{array}$ & $\begin{array}{l}A(1) \\
K(3) \\
H(1)\end{array}$ & $\begin{array}{l}\mathrm{K}(3) \\
\mathrm{S}(1) \\
\mathrm{V}(1)\end{array}$ & $\begin{array}{l}\mathrm{A}(1) \\
\mathrm{K}(2) \\
\mathrm{V}(2)\end{array}$ & $\begin{array}{l}\mathrm{A}(2) \\
\mathrm{K}(3)\end{array}$ & $\begin{array}{l}A(2) \\
K(1)\end{array}$ \\
\hline E-Modul & 10.400 & 11.400 & 10.300 & 11.500 & 12.700 & 12.200 & 11.100 & 13.100 \\
\hline Variations.-koeff. & 5,9 & 4,2 & 1,7 & 5,0 & 4,5 & 2,3 & 3,6 & 0,9 \\
\hline $\begin{array}{l}\text { A: Ast } \\
\text { K: Keilzinkenverbindung } \\
\text { H: Holz } \\
\text { 1) unterste Holzlamelle }\end{array}$ & $\begin{array}{l}\text { S: Schub } \\
\text { V: Versuc }\end{array}$ & $\begin{array}{l}\text { versager } \\
\text { hsabbru }\end{array}$ & & & & & & \\
\hline
\end{tabular}

In Bild 4 sind die Mittelwerte der Traglasten für das Versagen der untersten Holzlamelle und des Trägers dargestellt. Aus Simulationsrechnungen wurde zusätzlich die Traglast für die unverstärkten Querschnitte ermittelt und ebenfalls in diesem Diagramm aufgetragen.

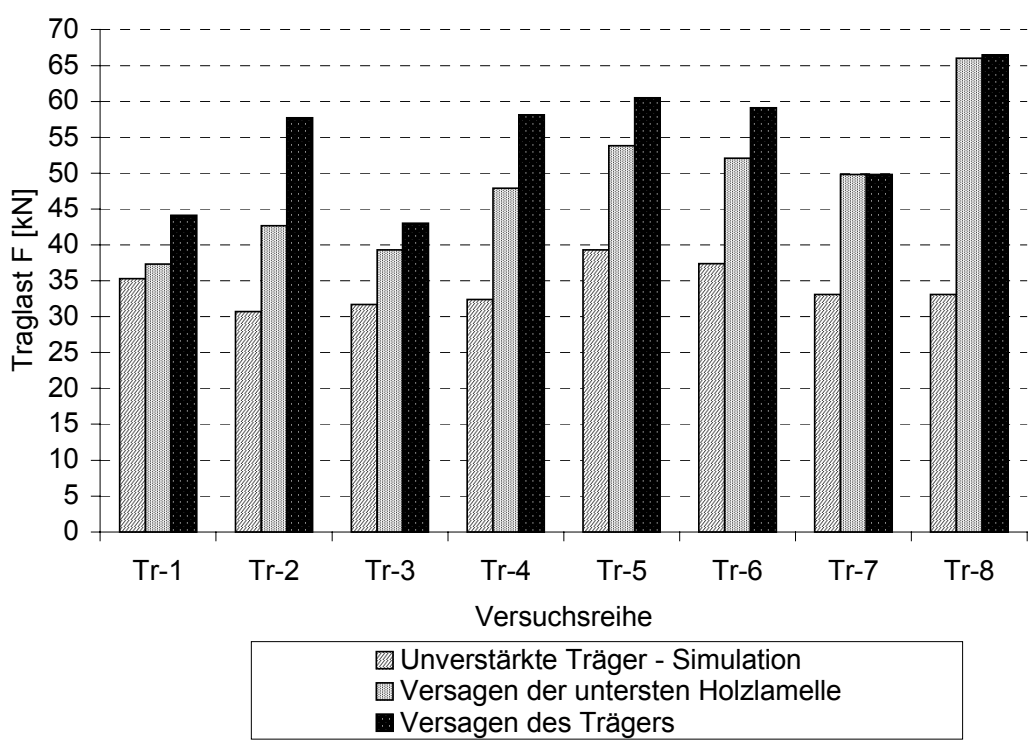

Bild 4: Mittelwerte der Traglast für das Versagen der Randlamelle und des Trägers

Fig. 4: Mean Values of the ultimate load for first and global failure

In Bild 5 ist ein Vergleich des Elastizitätsmoduls aus den Versuchen und nach einer Berechnung mit der Verbundtheorie für unverstärkte und verstärkte Querschnitte dargestellt. Aus der Berechnung ergibt sich für die verstärkten Querschnitte eine gute Übereinstimmung mit den Versuchsergebnissen. Der tendenziell höhere Elastizitätsmodul aus der Berechung der Träger im Vergleich zu den im Versuch ermittelten Werten, lässt sich durch den höheren Elastizitätsmodul bei dynamisch ermittelten Moduln gegenüber statisch ermittelten Werten der Einzelbretter erklären. Die Eingangswerte dieser Berechnung bestehen aus den Längsschwingungs-E-Moduln, die bei der Holzauswahl für jedes Brett ermittelt wurden. Weiterhin ist zu erkennen, dass eine Erhöhung der Biegesteifigkeit im Vergleich zu unverstärkten Querschnitten vorhanden ist. Aus einem Vergleich der 
einzelnen Versuchsreihen lässt sich entnehmen, dass ein Einfluss der Lage und Verstärkungsmenge der FVK-Lamelle deutlich erkennbar ist.

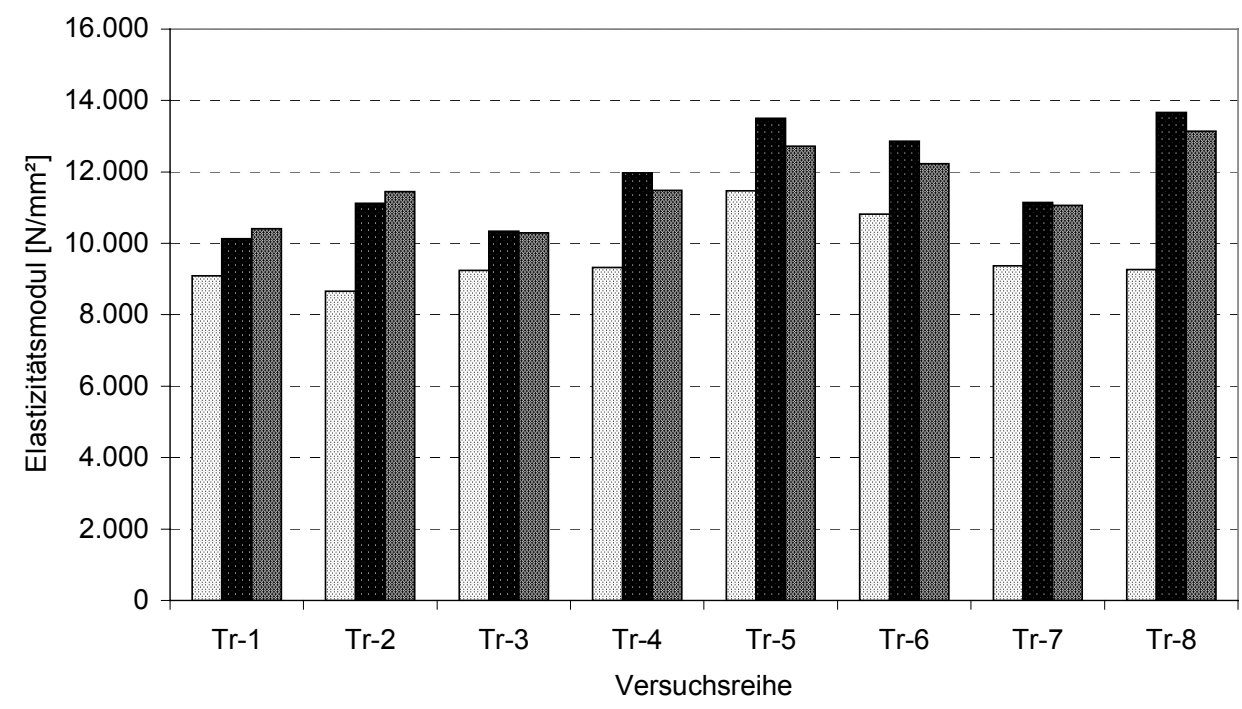

国echnung - unverstärkt $\mathbf{0}$ Rechnung - verstärkt 술 Versuch nach DIN EN 408

Bild 5: Mittelwerte des Elastizitätsmoduls aus den Versuchen und berechnete Werte des Elastizitätsmoduls für die unverstärkten und verstärkten Querschnitte

Fig. 5: Mean values of the MOE from the tests and calculated values of MOE for not reinforced and reinforced girders

\subsubsection{Tragverhalten}

Insgesamt traten bei den Bauteilversuchen vier verschiedene Versagensarten auf. Das Tragverhalten war dabei abhängig vom Anteil der Verstärkung am Gesamtquerschnitt, von der Qualität der Klebefuge, der Lage der FVK-Lamelle und den Materialeigenschaften der FVK-Lamelle und des BS-Holzes.

Bei der ersten Versagensart versagten die Bauteile ohne Ankündigung. Für die Versuchsreihen Tr-7 und Tr-8 war dies ein typisches Versagen, da bei diesen Versuchsreihen keine Randlamelle vorhanden war. In Bild 6 ist das Last-Verformungsdiagramm eines solchen Versuches dargestellt. Bei einzelnen Versuchen trat dieses Versagen auch bei Versuchsreihen mit Randlamelle auf. Bei diesen Versuchen war in der Regel nur eine geringe Krümmung der Last-Verformungskurve zu erkennen. 


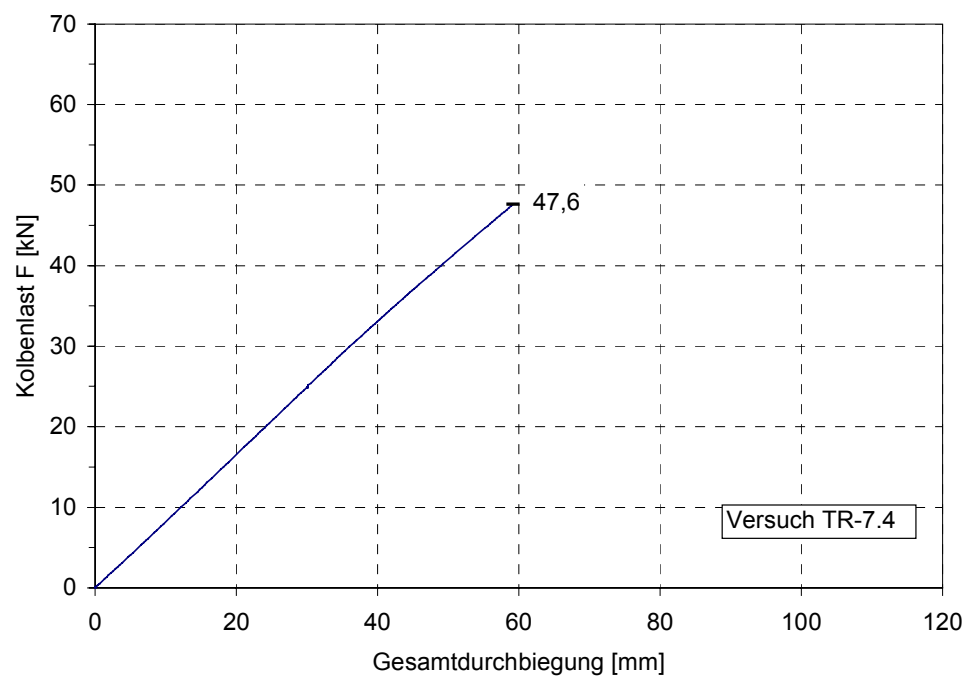

Bild 6: Last-Verformungsdiagramm des Versuchs Tr-7.4

Fig. 6: Load-deflection diagram of test Tr-7.4

Eine weitere Versagensart war ein Versagen der Randlamelle bei anschließender erneuter Lastaufnahme, ohne die zuvor erreichte Last wieder zu erreichen. In Bild 7 ist das Last-Verformungsdiagramm des Versuchs Tr-3.3 dargestellt, bei dem nach zweimaligem Lastabfall ein Versagen des Gesamtsystems unterhalb der Höchstlast erfolgte.

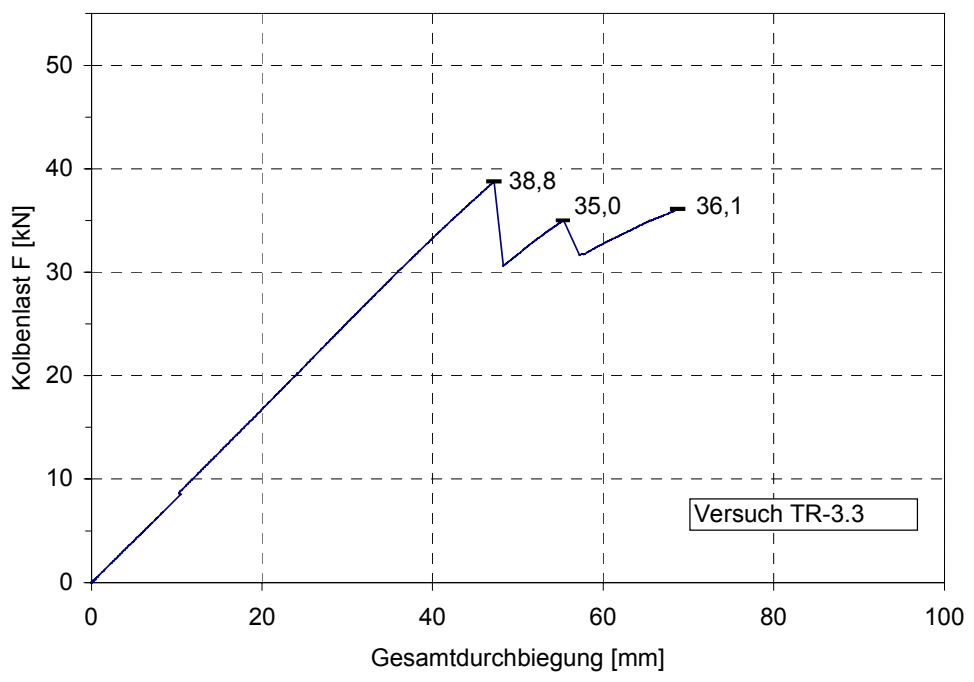

Bild 7: Last-Verformungsdiagramm des Versuchs Tr-3.3

Fig. 8: Load-deflection diagram of test Tr-3.3

Die dritte Versagensart ist in Bild 8 dargestellt. Nach dem Versagen der Randlamelle kam es zu einer weiteren Steigerung der Last, das Gesamtversagen erfolgte dann durch ein Versagen einer Holzlamelle oberhalb der FVK-Lamelle. 


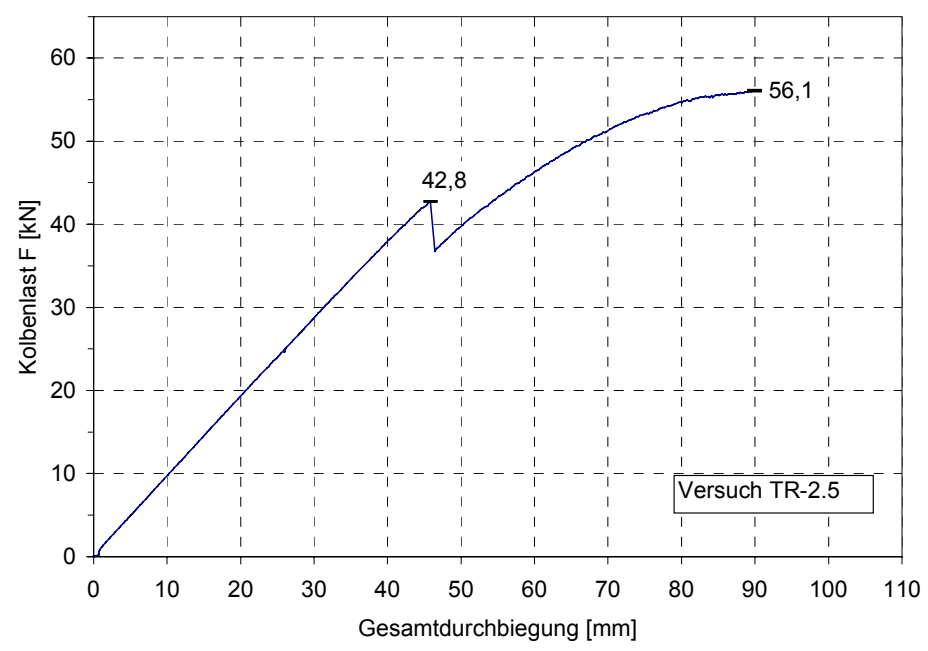

Bild 8: Last-Verformungsdiagramm des Versuchs Tr-2.5

Fig. 9: Load-deflection diagram of test Tr-2.5

In Bild 9 ist die vierte Versagensart dargestellt, bei der es zu einem stufenweisen Versagen kam. Dieses Versagen trat bei der Versuchsreihe Tr-5 und Tr-6 auf. Bei einigen Versuchen wurden die Versuche ohne ein Gesamtversagen des Bauteils abgebrochen. Nach Rissen in der Randlamelle und der zweiten bzw. dritten Holzlamelle von unten traten sehr große Druckfalten auf. Das Bauteil verformte sich ausgesprochen plastisch. Das Versagen erfolgte in mehreren Stufen, teilweise trat mehrfaches Versagen innerhalb einer Brettlamelle auf. In Bild 11 ist der zum LastVerformungsdiagramm in Bild 9 gehörige Querschnitt bei Versuchsabbruch dargestellt.

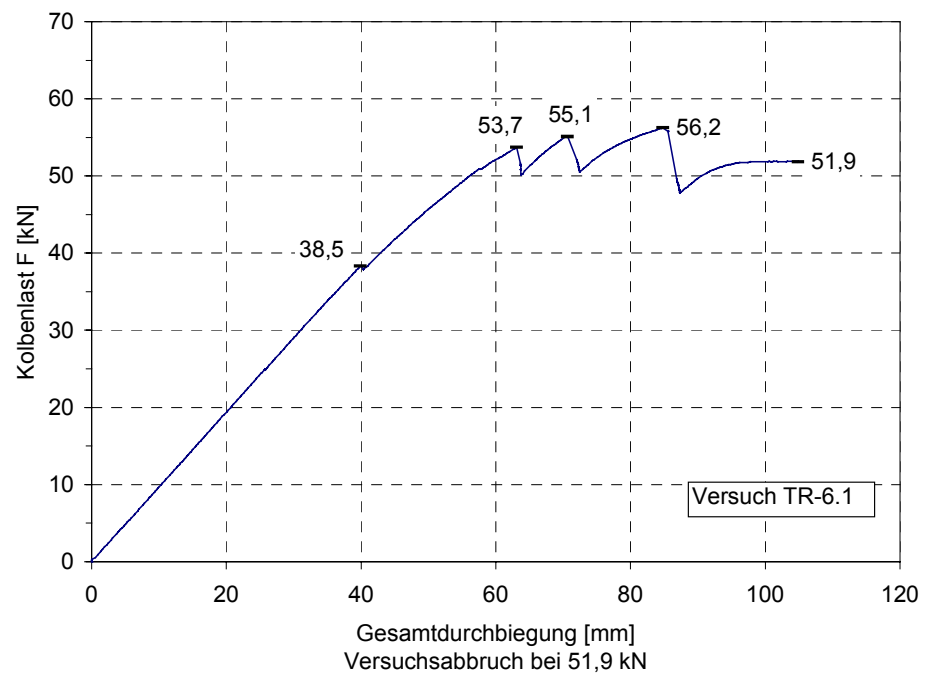

Bild 9: Last-Verformungsdiagramm des Versuchs Tr-6.1

Fig. 10: Load-deflection diagram of test Tr-6.1

Bis zum ersten Versagen einer Holzlamelle zeigte sich bei den meisten Versuchen keine bzw. nur eine geringe Krümmung der Last-Verformungskurve. Plastische Verformungen traten demnach bei diesen Versuchen nicht oder nur in einem geringem Umfang auf. Trat nach dem ersten Versagen kein Versagen des 
Gesamtsystems auf, so konnte teilweise ein ausgeprägt plastisches Verhalten beobachtet werden. In Bild 8 ist ein Beispiel mit einem durchschnittlichen plastischen Verhalten dargestellt. In einzelnen Versuchen wurden im Querschnitt Druckfalten bis zu etwa 1/3 der Querschnittshöhe beobachtet. Ein Beispiel einer solch stark ausgeprägten Druckfalte ist in Bild 11 dargestellt.

In Bild 10 ist das Tragverhalten des Versuchs Tr-2.5 mit einer Randlamelle und des Versuchs Tr-7.4 ohne Randlamelle darstellt. Die dazugehörigen Last-Verschiebungsdiagramme sind in Bild 6 und Bild 8 dargestellt. Beim Versuch Tr-2.5 war zunächst ein Versagen der Randlamelle zu beobachten. Im Anschluss erfolgte ein Ablösen der Randlamelle, bis es zu einem Versagen des Trägers ausgehend von den Holzlamellen oberhalb der zwei FVK-Lamellen kam. Die Biegesteifigkeit nahm dabei nach dem Versagen der Randlamelle kontinuierlich ab. Im Gegensatz hierzu verhielt sich der Prüfkörper Tr-7.4 bis zum Versagen, das ebenfalls von den Holzlamellen oberhalb der FVK-Lamelle ausging, fast bis zur Bruchlast linearelastisch.

Versuch Tr-2.5

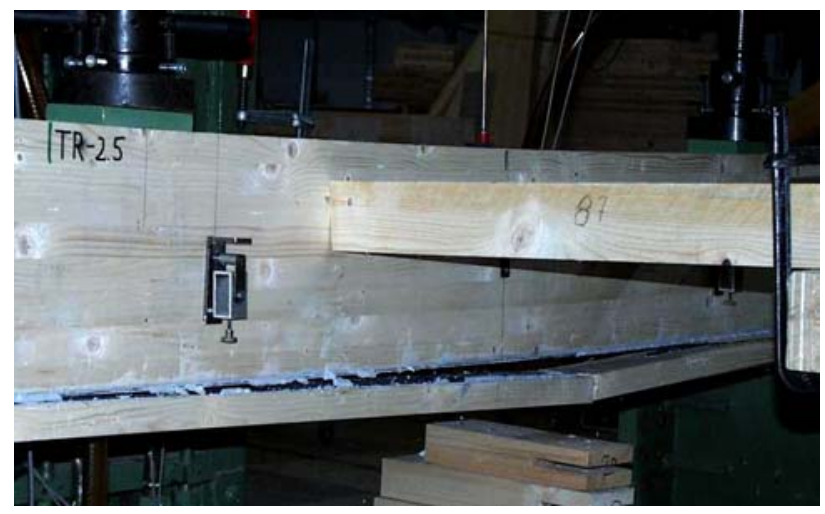

Versagen der Randlamelle und Ablösen

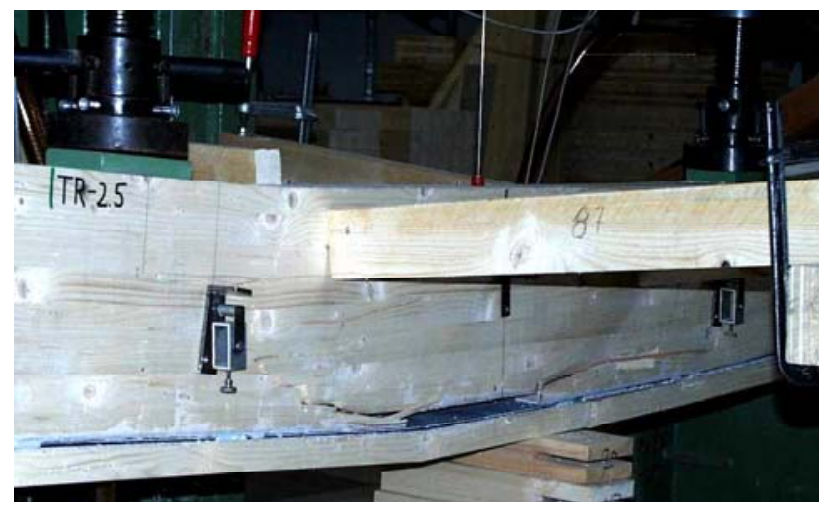

Gesamtversagen ausgehend von der Holzlamelle oberhalb der FVK-Lamelle
Versuch Tr-7.4

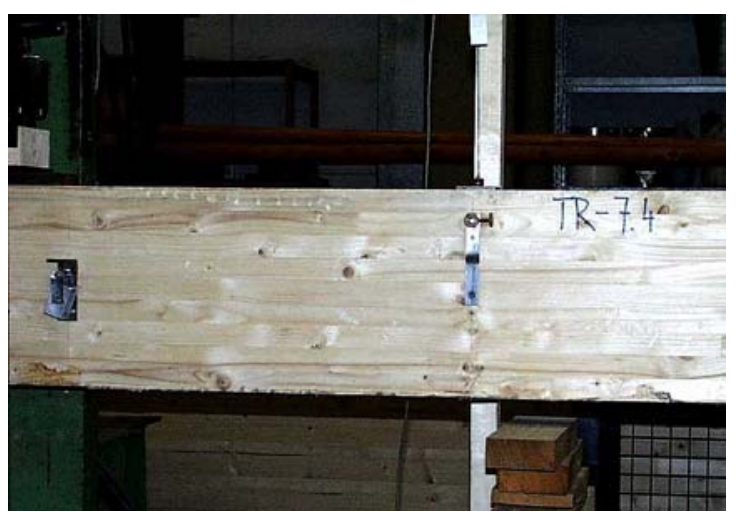

bei ca. $50 \%$ der Traglast

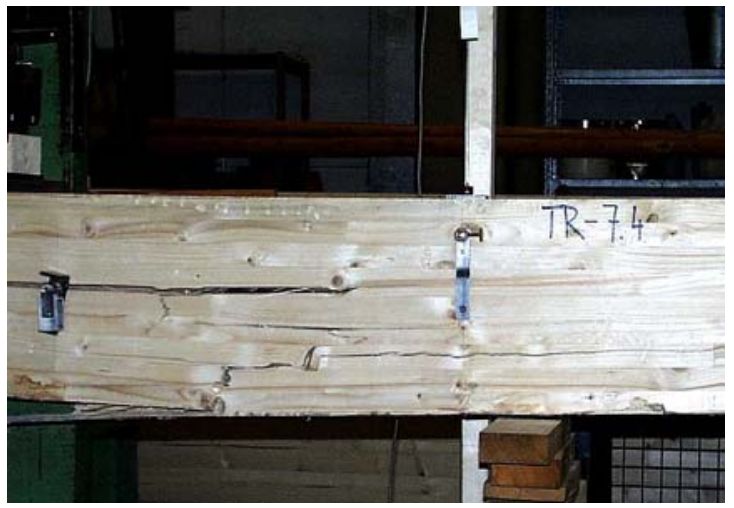

Gesamtversagen ausgehend von der Holzlamelle oberhalb der FVK-Lamelle

Bild 10: Bruchverlauf des Versuchs Tr-2.5 mit Randlamelle (links) und des Versuchs Tr-7.4 ohne Randlamelle (rechts)

Fig. 10: Progression of failure of the test Tr-2.5 with a lower timber lamella (left) and test Tr-7.4 without a lower timber lamella (right) 
Ein Gesamtversagen eines Versuchskörpers aufgrund eines Versagens der FVK-Lamelle erfolgte bei keinem Versuch. Die Zugfestigkeit der FVK-Lamellen wurde somit bei keiner Versuchsreihe ausgenutzt. Bei Versuchen mit mehreren Lagen von FVK-Lamellen wurden sowohl bei dem Epoxidharzklebstoff, als auch beim Polyurethanklebstoff, keine Ablöseerscheinungen bzw. ein Versagen der Klebefuge zwischen den einzelnen Lagen der FVK-Lamellen festgestellt. In Bild 11 ist der Ausschnitt des Prüfkörpers Tr-6.1 mit einer ausgeprägten Druckfalte bei versagter Randlamelle dargestellt. Die Randlamelle weist eine geringere Ablösung auf, als die in Bild 10 für den Prüfkörper Tr-2.5 dargestellt wird.

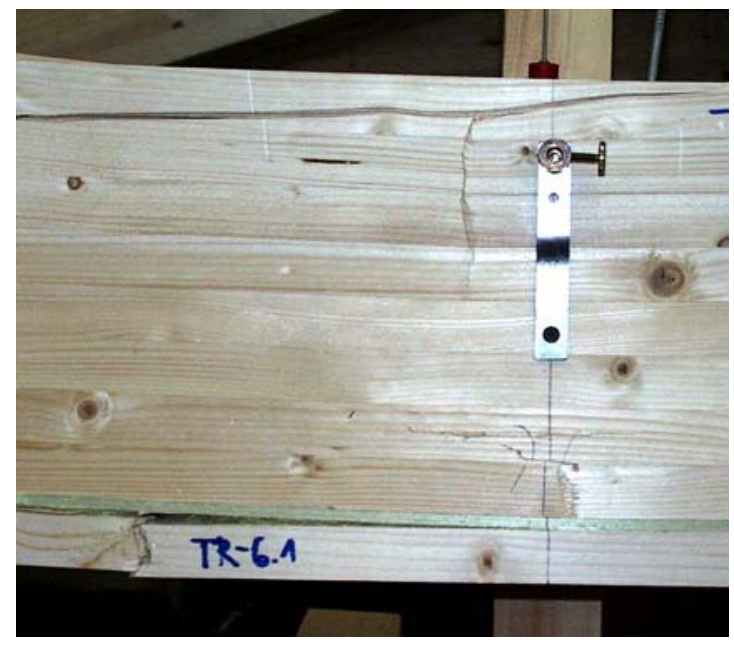

Bild 11: Ausbildung einer Druckfalte und geringes Ablöseverhalten der Randlamelle

Fig. 11: Development of a compression wrinkle and peeling of the adjacent timber lamination

\section{Anwendungsbereiche}

Die Kosten für AFK- und CFK-Lamellen sind derzeit trotz beständig sinkender Preise noch verhältnismäßig hoch. Dennoch kann eine Verstärkung von BS-Holz mit FVKLamellen aus Kostengründen gegenüber anderen Werkstoffen oder unverstärkten BS-Holz Trägern interessant sein.

Für die Verwendung von FVK verstärkten BS-Holz Trägern sind zwei Anwendungsbereiche zu unterscheiden. Die Applikation der FVK-Lamellen kann nachträglich für einen Holzträger eines bestehenden Tragsystems erfolgen, der aufgrund einer Nutzungsänderung bzw. einer fehlerhaften Auslegung des Bauteils aus technischen bzw. wirtschaftlichen Gründen nicht ersetzt werden kann. Als Klebstoffe eignen sich hierfür z.B. Epoxidharzklebstoffe. Diese Klebstoffe benötigen im Gegensatz zu den bei der BS-Holz Herstellung verwendeten Polyurethan- und Resorinharzklebstoffen keinen Pressdruck. Ein Pressdruck ist am bestehenden Tragsystem meist nur schwierig aufzubringen. Dies ermöglicht eine schnelle und einfache Applikation der FVK-Lamellen. Die Kostenvorteile bei der nachträglichen Verstärkung liegen im Vergleich zum Austausch des Bauteils in den geringeren Lohnkosten und in der Materialersparnis für einen entsprechend dimensionierten 
unverstärkten Träger, der bei einem Austausch des Bauteils eingebaut werden müsste.

Eine weitere Möglichkeit besteht in der Berücksichtigung FVK verstärkter BSHolz Träger bereits während der Planung eines Tragwerkes. Gründe hierfür kann eine erforderliche Beschränkung der Abmessungen des Bauteils sein oder die Aufnahme von Beanspruchungsspitzen durch eine partielle Verstärkung in Trägerlängsrichtung. Ein typisches Beispiel einer partiellen Verstärkung ist in Bild 12 dargestellt. Eine gezielte partielle Verstärkung führt zu einer Ausnutzung der Tragfähigkeit an verschiedenen Stellen des Tragsystems bei gleichbleibender Querschnittshöhe. Als Klebstoff bietet sich für dieses Anwendungsgebiet ein Klebstoff an, der in der Herstellung von BS-Holz ohnehin Verwendung findet wie z.B. der in der dargestellten Untersuchung verwendete Polyurethanklebstoff. Dies ermöglicht eine Produktion, die weitgehend mit der Herstellung von üblichem BSHolz identisch ist. Die Eignung eines entsprechenden Klebstoffes sollte allerdings nachgewiesen werden. Die Planung von FVK verstärkten BS-Holz vor dem Einbau in ein Tragwerk ermöglicht die Verstärkung auf eine einfache Art und Weise und kann visuell unauffällig im Querschnitt erfolgen. Durch eine Randlamelle aus Holz kann die FVK-Lamelle zudem gegen Feuer und ggf. gegen UV-Strahlung geschützt werden. Die Kostenvorteile dieser Verstärkungsvariante liegen in der Materialersparnis und in der Verringerung von Transport- und Einbaukosten, da bei den FVK verstärktem BSHolz Trägern aufgrund der geringeren Querschnittsabmessungen Holzvolumen eingespart wird und das Gewicht der Bauteile geringer ist.

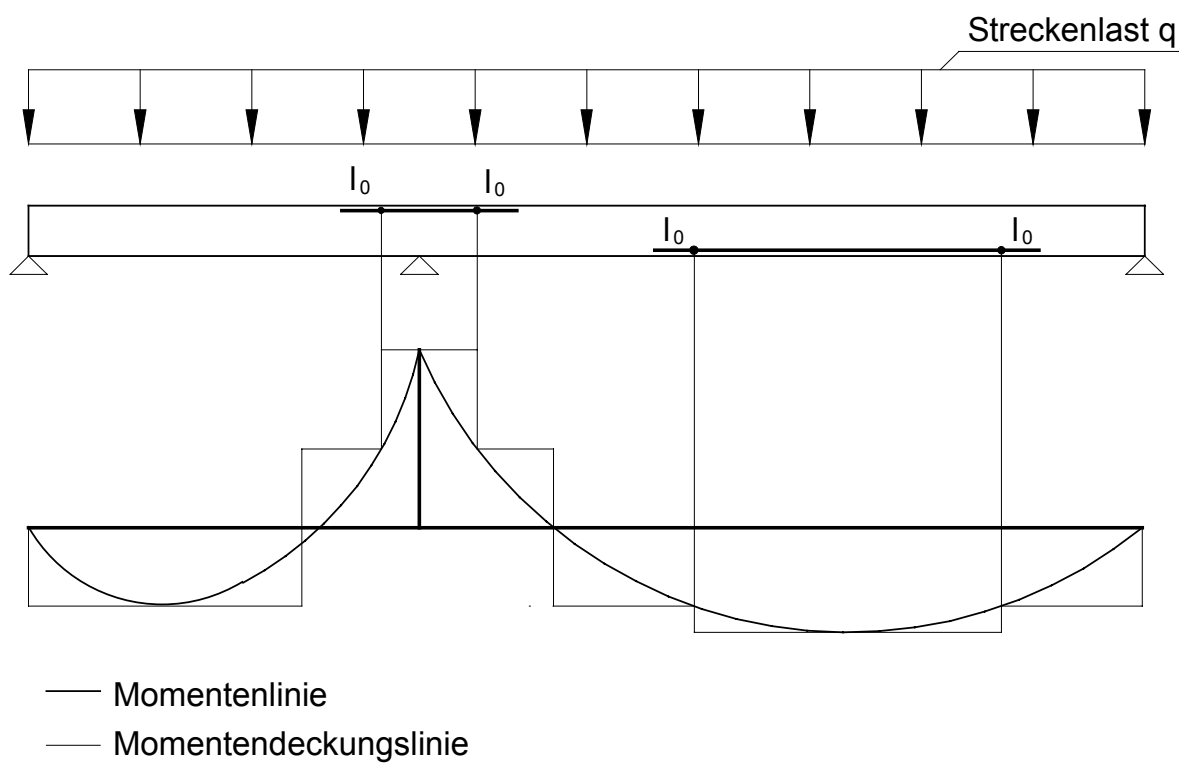

Bild 12: Beispiel einer partiellen Verstärkung an einem Durchlaufträger

Fig. 12: Example of a partial reinforcement of a continuous beam

Bei den Bauteilversuchen mit mehr als einer FVK-Lamellenlage wurde bei keinem der Versuchskörper ein Versagen der Klebefuge zwischen den einzelnen Lagen von FVK-Lamellen beobachtet, so dass auch eine Verklebung der FVK- 
Lamellen in mehreren Lagen mit den gewählten Klebstoffen möglich ist und der Verstärkungsgrad dem Tragsystem angepasst werden kann.

In der Regel wird durch die Verstärkung mit FVK-Lamellen eine Erhöhung der Tragfähigkeit angestrebt. Ziel der Verstärkung kann aber auch eine Erhöhung der Biegesteifigkeit sein. Die zuvor dargestellten Untersuchungsergebnisse zeigen allerdings, dass eine Erhöhung der Biegesteifigkeit nicht in demselben Maße erfolgt wie die Erhöhung der Tragfähigkeit. Im Vordergrund der Verstärkung sollte somit die Erhöhung der Tragfähigkeit stehen.

\section{Zusammenfassung}

Die Ergebnisse der Untersuchung zeigen, dass eine Erhöhung der Biegesteifigkeit und der Tragfähigkeit durch das Verstärken von BS-Holz Trägern mit FVK-Lamellen in der Zugzone der Querschnitte erfolgen kann. Die Erhöhung der Tragfähigkeit durch das Verstärken der Zugzone eines Biegeträgers ist ausgeprägter als die Erhöhung der Biegesteifigkeit. Eine Berechnung der Biegesteifigkeit nach der Verbundtheorie ergab eine gute Übereinstimmung mit den in Versuchen ermittelten Werten. Die Erhöhung ist sowohl für die Tragfähigkeit als auch für die Biegesteifigkeit vom Anteil der Verstärkungsmenge am Gesamtquerschnitt, den Materialeigenschaften der FVK-Lamellen und des Holzes und der Lage der FVKLamellen im Querschnitt abhängig.

Bei Versuchsreihen mit einer Randlamelle aus Holz unterhalb der FVK-Lamelle wurde bei den Versuchsreihen mit CFK-Lamellen ein deutliches Ablösen der FVKLamellen nach dem Versagen der Randlamelle festgestellt. Dieser Effekt reduziert die Tragfähigkeit in dem abgelösten Bereich. Bei den Versuchsreihen mit der AFKLamelle wurde ein derart ausgeprägtes Ablösen der Randlamelle nicht beobachtet. Dies hatte bei einigen Versuchen dieser Versuchsreihen zur Folge, dass es in der selben Brettlamelle, an verschiedenen Stellen in Trägerlängsrichtung, zu einem Versagen der jeweiligen Brettlamelle kam. Dies zeigte sich auch durch einen stufigeren Verlauf der Last-Verformungsdiagramme. Bei einer Reduzierung des Ablöseeffektes sind zusätzliche Steigerungen der Tragfähigkeit zu erwarten.

Bei Klebefugen zwischen den einzelnen FVK-Lamellen konnten weder beim Epoxidharzklebstoff noch beim Polyurethanklebstoff ein Versagen festgestellt werden. Ein Versagen der Klebefuge konnte nur zwischen Holz und FVK-Lamelle, ausgelöst durch das Gesamtversagen bzw. das bereits beschriebene Ablösen, festgestellt werden. Bei einigen der Versuchsreihen mit einem hohen Verstärkungsgrad konnten sichtbare Druckfalten bis zu 1/3 der Querschnittshöhe beobachtet werden.

Das Verstärken von BS-Holz Trägern ist mit im Holzbau gängigen Klebstoffen und Herstellungsverfahren möglich und bietet die Möglichkeit, signifikante Steigerungen der Tragfähigkeit und Biegesteifigkeit zu erzielen. 


\section{Literatur}

Blaß HJ, Romani M (2000) Trag- und Verformungsverhalten von Verbundträgern aus Brettschichtholz und faserverstärkten Kunststoffen. Forschungsbericht der Versuchsanstalt für Stahl, Holz und Steine, Abt. Ingenieurholzbau der Universität Karlsruhe 